\title{
Amelioration of Bile Duct Ligation Induced Liver Injury by Lactoferrin: Role of Nrf2/HO-1 Pathway
}

\author{
Aya R. Ayob ${ }^{1}$, Aya H. Al-Najjar², * and Azza S. Awad ${ }^{2}$
}

\begin{abstract}
${ }^{1}$ Department of Pharmacology and Toxicology, Faculty of Pharmacy, $6^{\text {th }}$ of October University, Giza, Egypt. ${ }^{2}$ Department of Pharmacology and Toxicology, Faculty of Pharmacy (Girls), Al-Azhar University, Cairo, Egypt.

*Correspondence: ayahesham.pharmg@azhar.edu.eg
\end{abstract}
Article history: Received: 2021-05-30
Revised: 2021-08-2
Accepted: 2021-08-9

\begin{abstract}
Cholestasis is one of the major determinants which causes hepatic injury and might lead to liver complications. In cholestasis, toxic bile acids accumulate with decrease in the capacity of detoxification. It can be stimulated experimentally by bile duct ligation (BDL) to block the bile flow from the liver. As for the treatment, lactoferrin (LF), the iron-binding glycoprotein, has been evaluated in various studies for the treatment of infections, inflammation, and cancer. This study aims to illustrate the curative effects of LF on BDL-induced hepatic injury in rats. Animals were randomly allocated into 4 groups: the first group is the control sham; the subjects of the second group have been subjected to a surgery of BDL; the latter procedure has been induced in the third group, 14 days later, they have been subjected to the treatment with $\mathrm{LF}$ ( $300 \mathrm{mg} / \mathrm{kg} / \mathrm{day}$, po) for two weeks; as for the last group, LF has been administered $(300 \mathrm{mg} / \mathrm{kg} / \mathrm{day}$, po) for two weeks. BDL elevated biomarkers of hepatocellular damage (aspartate aminotransferase (AST) and alanine aminotransferase (ALT)) and obstructive cholestatic injury (gamma-glutamyl transferase ( $\gamma$-GT) and total bilirubin). In addition, it provoked oxidative stress manifested by increase in hepatic MDA and reduction of SOD with down-regulation of Nrf2/HO-1 pathway. LF treatment ameliorated these effects through its antioxidant activity by activation of Nrf2/HO-1 pathway that leads to decrease in hepatic MDA and elevation of SOD. In conclusion; lactoferrin alleviated hepatic injury induced by BDL via activating Nrf2 gene expression that might be led back to its antioxidant properties.
\end{abstract}

Keywords: Cholestasis; Lactoferrin; Bile Duct Ligation (BDL); Nrf2; Oxidative stress.

\section{INTRODUCTION}

Liver is the main vital organ in the body that has several important functions, as metabolism of ingested elements and toxic agents detoxification ${ }^{1}$. Hepatic injury may be caused by different agents such as cholestasis, which involves a wide spectrum of harmful diseases and may lead to complications of the liver ${ }^{2}$.

Cholestatic liver damage is one of the main causes of hepatic complications, just as it is in chronic liver disease patients. Models have been developed experimentally to resemble different aspects of the disease $^{3,4,5}$. One of the most essential experimental models is the surgical BDL, which is utilized to generate obstructive cholestatic injury in rats and mice ${ }^{4,6}$.
Blocking of bile flow through the liver and accumulation of bile acids leads to stimulation of genes referred to oxidative stress, such as nuclear factor erythroid 2-related factor 2 (Nrf2) protein in hepatocytes ${ }^{7}$.

The injury may be generated by the action of bile acids through reactive oxygen species (ROS) ${ }^{8}$.

According to available guidelines, Ursodeoxycholic acid (UDCA) is regarded as the first-line treatment for primary biliary cholestasis ${ }^{10}$. But, many patients cannot respond to UDCA and may need liver transplantation ${ }^{11}$. So, there is a need for another curative agent for the management of cholestatic liver injury.

The iron-binding glycoprotein, lactoferrin (LF), is one of the transferrin family and exists in exocrine

Cite this article: Ayob, A., Al-Najjar, A., Awad, A. Amelioration of Bile Duct Ligation Induced Liver Injury by Lactoferrin: 84 Role of Nrf2/HO-1 Pathway. Azhar International Journal of Pharmaceutical and Medical Sciences, 2021; 1 (3): 84-90. doi: 10.21608/aijpms.2021.78232.1076 
secretion, serum and neutrophils ${ }^{12,13 .}$ LF can defend against different infections and has a significant role in immune responses. It shows antimicrobial actions against bacterial and parasitic infections in addition to its anticancer activity ${ }^{12,14}$. Therefore, LF has been reported to have a broad scale of biological activities, involving anti-inflammatory, anticancer, antimicrobial, antioxidant, and immunomodulatory-enhancing effects ${ }^{15,} 16$.

The use of LF has been reported in the treatment of different diseases as hepatitis $\mathrm{C}$ virus infections ${ }^{17}$, osteoporosis $^{18}$, and cancer ${ }^{19}$.

The present study examined the role of lactoferrin in experimental bile duct ligation induced liver injury. We showed its crucial role in amelioration of cholestatic liver injury mainly caused by oxidative stress.

\section{MATERIALS AND METHODS}

\subsection{Materials}

\subsubsection{Animals}

Adult male Sprague-Dawley rats (7-8 weeks) with a weight range of (180-220 g) were used in this study. Rats were purchased from the National Research Center animal house (Giza, Egypt). They were kept in a controlled condition of the temperature $\left(25^{\circ} \mathrm{C}\right)$ with a 12-hour light/dark cycle. They were given regular rat chow in addition to tap water ad libitum. This study was done as stated by the Ethics Committee of the faculty of Pharmacy Al-Azhar University, Egypt (permit number: 229/2019) to ensure the welfare of the animals throughout the experiment.

\subsubsection{Drugs}

Lactoferrin powder (Hygint Pharmaceuticals) was used and dissolved in saline. Ketoprofen was purchased from Amriya Pharm. IND. (Alexandria, Egypt). Pentobarbital was purchased from SigmaAldrich (Missouri, United States).

\subsection{Methods}

\subsubsection{The Experimental design}

Surgical bile duct ligation (BDL) is a common experimental model for acute cholestasis ${ }^{20}$. Rats were randomly distributed into 4 groups ( 6 animals/ group): 1) Sham, 2) $\mathrm{BDL}, 3) \mathrm{BDL}+\mathrm{LF}$ $(300 \mathrm{mg} / \mathrm{kg} / \mathrm{day}, \mathrm{po})^{21}$ and 4) LF $(300 \mathrm{mg} / \mathrm{kg} / \mathrm{day}$, po). The surgery was done by opening the abdominal cavity; also the common bile duct was isolated without ligation in both sham and LF groups. While, the common bile duct was ligated in BDL and BDL+LF groups as described in $^{5}$. The anesthesia was done by pentobarbital (50 mg/kg/ip) with application of ophthalmic ointment to inhibit dryness of the cornea. The proximal and distal sections of the common bile duct were doubleligated by silk sutures (4-0). Rats were administrated ketoprofen (3 $\mathrm{mg} / \mathrm{kg} / \mathrm{im}$ ) for analgesia after surgery ${ }^{22}$ and saline was injected subcutaneously for compensation of fluid loss during surgery. Then, rats were kept in a warm condition till they were completely awake and active. After total BDL; fourteen days were enough to induce liver injury, according to the study of Tag et al., $2015^{5}$, so LF treatment was started after 14 days, so the rats in groups LF and BDL+LF received LF (300mg/kg/day, po) for two weeks.

Rats were weighed and placed under light anesthesia at the end of the experiment, and blood was drawn from the retro-orbital puncture to assess biochemical parameters. Cervical dislocation was done to euthanize the rats, and the entire liver was separated.

The liver tissue was divided into two sections: one part was homogenized in phosphate-buffered saline (50mM K2HPO4, pH 7.5) for ELISA experiments. The other tissue part was fixed in RNAlater ${ }^{\circledR}$ (Qiagen) overnight at $4{ }^{\circ} \mathrm{C}$, and after that was frozen at $80{ }^{\circ} \mathrm{C}$ for the experiment of qPCR.

\subsubsection{Measurement of liver functions}

Serum activities of liver enzymes aspartate aminotransferase (AST), alanine aminotransferase (ALT), and gamma-glutamyl transferase $(\gamma-G T)$ with total bilirubin were measured using commercial kits (MyBioSource, San Diego, USA) according to the manufacturer's protocol.

\subsubsection{Assessment of oxidative stress markers}

Malondialdehyde (MDA) was measured as an indication for hepatic lipid peroxidation by using thiobarbituric acid (Chemie Gmbh, Steinheim, Germany) ${ }^{23}$. The inhibitory effect of superoxide dismutase (SOD) enzyme on the reduction of nitro blue tetrazolium dye was used to determine SOD enzyme activity ${ }^{24}$.

\subsubsection{Quantitative real time PCR ( $q P C R)$}

Extraction of total RNA from liver tissue was done using a kit provided by (Thermo Fisher Scientific Inc. Germany.) as the manufacturer's provided steps. Quantitative real time PCR of the studied genes: The kit was provided by Vivantis, ViPrimePLUS One Step Taq RT-qPCR Green 
Master Mix I with ROX (SYBR Green Dye) then the $\Delta \Delta \mathrm{Ct}$ method was used to calculate the relative gene expression. The Primer sequences: Forward primer: TCCCAAACAAGATGCCTTGT, Reverse primer: AGAGGCCACACTGACAGAGA. The gene bank accession number is NM_031789.2.

\subsubsection{Assessment of total tissue protein}

Total proteins were determined by using a reagent kit (Biodiagnostic Company, Giza, Egypt) in the manner of the manufacturer's protocol.

\subsubsection{Statistical analysis}

Data expression was performed as means \pm SD. For statistical analysis; one-way analysis of variance (ANOVA) followed by Tukey- multiple comparisons test $(\mathrm{p}<0.05)$ was used. GraphPad prism software (version 5) (GraphPad Software Inc., USA) was utilized and a probability level of less than 0.05 was believed to be statistically significant

\section{RESULTS}

\subsection{Effect of LF on hepatocytes integrity}

The serum AST and ALT activities of BDL rats were markedly increased by $658 \%$ and $831 \%$, respectively, when compared to the sham group (Figure 1). This indicates a damage of hepatocytes induced by BDL. Additionally, the cholestasis main markers were increased by BDL which illustrated by the elevation in $\gamma$-GT plus total bilirubin by $779 \%$ and $1327 \%$, respectively, when compared with the sham group (as demonstrated in Figure 1).

When compared with the sham rats, LF administration reduced the growth of AST and ALT by $263 \%$ and $318 \%$, respectively (Figure 1). In addition, cholestatic injury was reduced because $\gamma$ GT plus total bilirubin were reduced by $207 \%$ and $339 \%$, respectively, as compared with the sham group. In sham rats, these hepatocellular integrity parameters were unaffected by LF administration.

\subsection{Effect of LF on markers of oxidative stress}

Bile duct ligation provoked oxidative stress which was indicated by a substantial rise in hepatic MDA by $1070 \%$ and a decrease in SOD by $1413 \%$ compared to the sham group, while LF treatment reduced MDA by $762 \%$ and elevated SOD by $164 \%$ compared with the sham group (Figure 2).
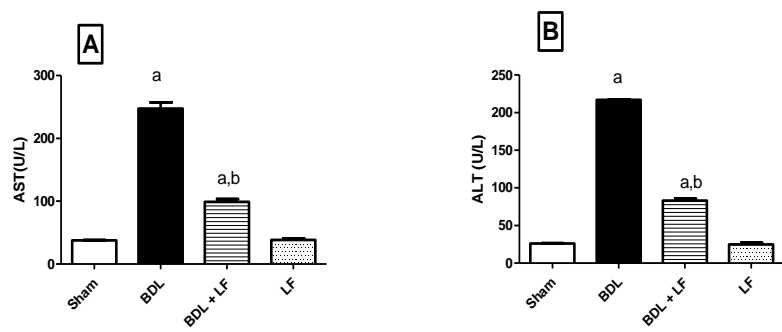

C

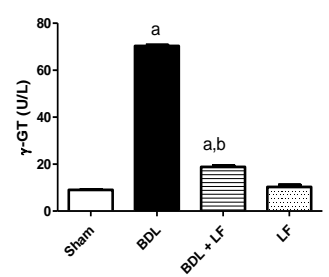

D

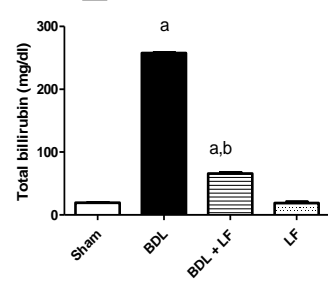

Figure 1(A-D): Effect of LF on liver biochemical parameters changes induced by bile-duct ligation (BDL). Surgical BDL was done and after 14 days LF (300 $\mathrm{mg} / \mathrm{kg} / \mathrm{day}$, orally) was given for two weeks.

(A) Aspartate transaminase (AST) serum activity, (B) alanine transaminase (ALT) serum activity, (C) gamma glutamyl transferase ( $\gamma$-GT) and (D) total bilirubin were then measured. Data are displayed as mean $\pm \operatorname{SD}(n=6) .{ }^{\text {a }}$ $\mathrm{p} \leq 0.05$ compared to the sham group, ${ }^{\mathrm{b}} \mathrm{p} \leq 0.05$ compared to BDL group. One-way ANOVA was used for statistical analysis, with Tukey Kramer as a post hoc measure.
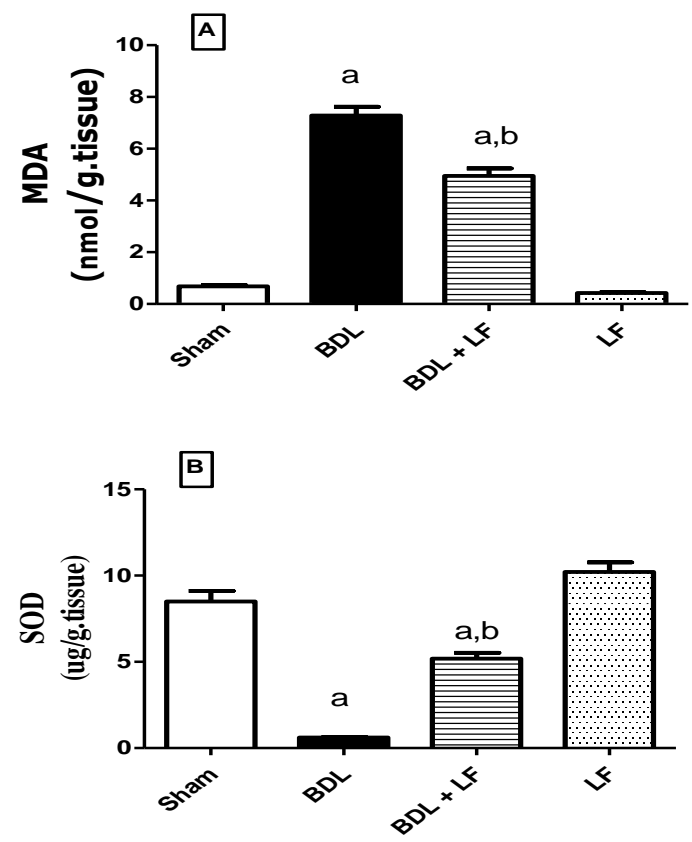

Figure 2 (A\&B): Impact of LF on oxidative stress induced by bile-duct ligation (BDL). Surgical BDL was done and after 14 days LF (300 mg/kg/day, orally) was administered for two weeks. (A) Hepatic 
Malondialdehyde (MDA) and (B) Superoxide dismutase (SOD).Values are displayed as mean $\pm \mathrm{SD}(\mathrm{n}=6)$. a $\mathrm{p} \leq$ 0.05 compared to the sham group, $\mathrm{b} p \leq 0.05$ compared to BDL group. One-way ANOVA was used for statistical analysis, with Tukey Kramer as a post hoc measure.

\subsection{Effect of LF on Nrf2/HO-1}

The antioxidant Nrf2 expression was downregulated by $\mathrm{BDL}$ to $76 \%$ as compared with the sham group while LF treatment up-regulated its hepatic expression by $252 \%$ as compared with the BDL group. In addition, HO- 1 content in liver tissue was significantly decreased in BDL rats by 6 fold when compared with rats in sham group, while LF treatment significantly elevated its content. LF treatment in the sham group showed no significant effect on Nrf2 expression or HO-1 content (Figure 3).

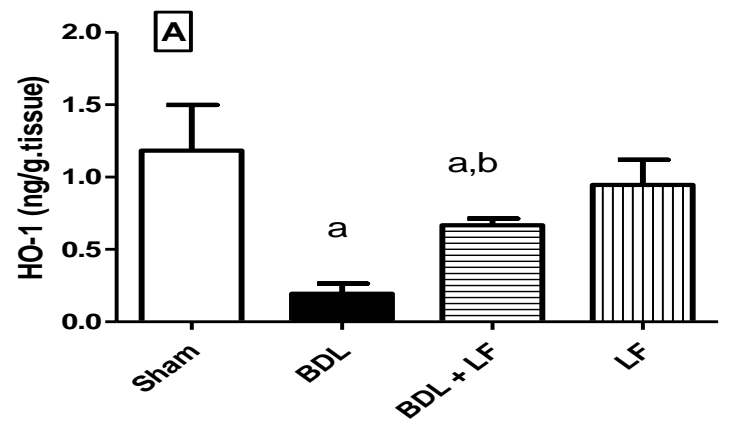

B

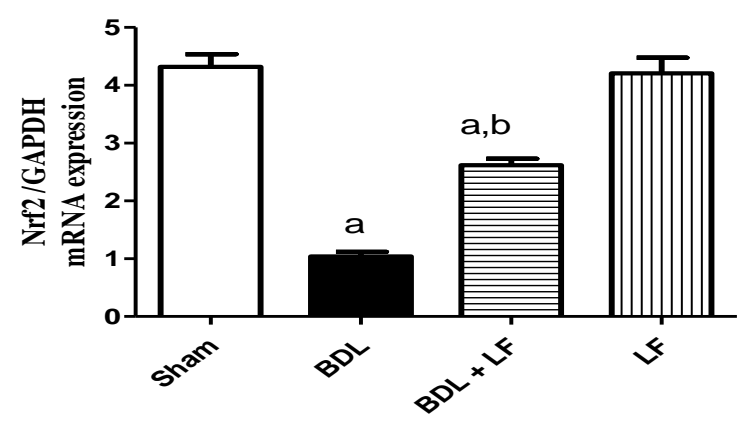

Figure 3 (A\&B): Effect of LF on hepatic Nrf2/HO-1 changes caused by bile-duct ligation (BDL). Surgical $\mathrm{BDL}$ was done and after 14 days $\mathrm{LF}(300 \mathrm{mg} / \mathrm{kg} / \mathrm{day}$, orally) was administered for two weeks. (A) Hemeoxygenase-1content (HO-1) in addition (B) Expression of Nuclear factor erythroid 2-related factor 2 (Nrf2), were then assessed. Values are illustrated as mean $\pm \mathrm{SD}(\mathrm{n}=6) .{ }^{\mathrm{a}} \mathrm{p} \leq 0.05$ compared to the sham group, ${ }^{\mathrm{b}} \mathrm{p} \leq$ 0.05 compared to BDL group. One-way ANOVA was used for statistical analysis, with Tukey Kramer as a post hoc measure.

\section{DISCUSSION}

Cholestasis is identified by accumulation of bile acids. It is one of the important caustic determinants for liver injury, which often progresses to other consequences of liver disease and may lead to morbidity and mortality ${ }^{25}$.

Surgical bile duct ligation is an important model in rats to cause cholestasis and then liver injury ${ }^{4,26}$.

Lactoferrin was reported by several studies to have different activities as antioxidant, antiinflammatory ${ }^{15,16}$ and hepatoprotective properties; as previous studies showed LF hepatoprotection against acetaminophen ${ }^{27}$ and liver injury caused by concanavalin $\mathrm{A}$ in mice ${ }^{28}$.

In this study, we researched the role of LF in amelioration of liver injury caused by BDL in a rat model.

The cellular integrity was affected and liver dysfunctions occurred by BDL as indicated by raised serum AST and ALT levels, also cholestasis was evidenced by increase of bilirubin as well as $\gamma$ GT, while treatment with LF ameliorates these effects. These results come in parallel with previous studies $^{29}$, 30. Furthermore, cholestasis leads to oxidative stress that showed by increase in hepatic MDA and reduction in SOD. BDL affected the antioxidant Nrf2 pathway, as it down-regulated gene expression of Nrf2 and decreased the HO-1 content. Former studies confirmed that BDL caused oxidative stress $^{30,31 .}$

The basic leucine zipper transcription factor, Nrf2, has been shown to protect multiple organs against harmful reactive chemicals such as chemical carcinogens $^{32}$. In oxidative stress conditions, Nrf2 is transferred to the nucleus and stimulates the transcription of various antioxidant genes such as HO-1 and SOD ${ }^{33}$. HO-1 is a powerful antioxidant and it is a downstream target factor of Nrf2 that increases hepatocyte survival. So Nrf2 translocation HO- 1 can activate HO- $1^{34}$.

In this study, treatment with LF inhibited oxidative stress by increasing SOD, an important antioxidant and detoxification enzyme of intracellular protective mechanisms, also investigated by a reduction in hepatic MDA level. Additionally, LF protected the liver by activation of $\mathrm{Nrf} / \mathrm{OH}$ 1 pathway.

\section{CONCLUSION}

In conclusion, this study illustrated that lactoferrin ameliorated the liver injury caused by BDL oxidative stress, possibly via its antioxidant activity by activation of Nrf2 gene expression. 
Funding: This research has obtained no funding from any source.

Acknowledgments: The authors are grateful to Hygint Company for supplying lactoferrin powder for free.

Conflicts of Interest: The authors declare no conflict of interest.

Ethical Statement: This study was done according to the Ethics Committee of the faculty of Pharmacy Al-Azhar University, Egypt (permit number: 229/2019). Unnecessary disturbance of animals, pressure and tough maneuver was avoided.

Author Contribution: All authors were hand by hand in each part of this research.

List of Abbreviations: $\gamma$-GT, gamma-glutamyl transferase; ALT, alanine aminotransferase; AST, Aspartate aminotransferase; BDL, bile duct ligation; HO-1, Hemeoxygenase- 1; LF, Lactoferrin; MDA, Malondialdehyde; Nrf2, nuclear factor erythroid 2-related factor 2; ROS, Reactive oxygen species; SOD, superoxide dismutase.

\section{REFERENCES}

1. Almeer RS, Alarifi S, Alkahtani S, Ibrahim SR, Ali D, Moneim A. The potential hepatoprotective effect of royal jelly against cadmium chloride-induced hepatotoxicity in mice is mediated by suppression of oxidative stress and upregulation of Nrf2 expression. Biomed Pharmacother. 2018;106:1490-8.

2. Gossard AA, Talwalkar JA. Cholestatic Liver Disease. Med Clin North Am [Internet]. 2014;98(1):73-85. Available from: https://www.sciencedirect.com/science/article/pii/ S0025712513001260.

\section{3- Liedtke C, Luedde T, Sauerbruch T, Scholten D,} Streetz K, Tacke F, et al. Experimental liver fibrosis research: update on animal models, legal issues and translational aspects. Fibrogenesis Tissue Repair. 2013;6(1):1-25.

4. Liedtke C, Luedde T, Sauerbruch T, Scholten
D, Streetz K, Tacke F, et al. Experimental liver fibrosis research: update on animal models, legal issues and translational aspects. Fibrogenesis Tissue Repair. 2013;6(1):1-25.

5. Tag CG, Sauer-Lehnen S, Weiskirchen S, Borkham-Kamphorst E, Tolba RH, Tacke F, et al. Bile duct ligation in mice: induction of inflammatory liver injury and fibrosis by obstructive cholestasis. J Vis Exp JoVE. 2015;(96):1-11.

6. Kountouras J, Billing BH, Scheuer PJ. Prolonged bile duct obstruction: a new experimental model for cirrhosis in the rat. $\mathrm{Br} \mathbf{J}$ Exp Pathol. 1984;65(3):305-311.

7. Allen K, Jaeschke H, Copple BL. Bile acids induce inflammatory genes in hepatocytes: a novel mechanism of inflammation during obstructive cholestasis. Am J Pathol. 2011;178(1):175-86.

8. Svegliati-Baroni G, Ridolfi F, Hannivoort R, Saccomanno S, Homan M, De Minicis S, et al. Bile acids induce hepatic stellate cell proliferation via activation of the epidermal growth factor receptor. Gastroenterology. 2005;128(4):1042-55.

9. Baarsma HA, Menzen MH, Halayko AJ, Meurs H, Kerstjens HAM, Gosens R. $\beta$-Catenin signaling is required for TGF- $\beta 1$-induced extracellular matrix production by airway smooth muscle cells. Am J Physiol Cell Mol Physiol. 2011;301(6):L956-65.

10. Lindor KD, Poupon R, Poupon R, Heathcote EJ, Therneau T. Ursodeoxycholic acid for primary biliary cirrhosis. Lancet. 2000;355(9204):657-8.

11. Kuiper EMM, Hansen BE, de Vries RA, den Ouden-Muller JW, Van Ditzhuijsen TJM, https://aijpms.journals.ekb.eg/ 
Haagsma EB, et al. Improved prognosis of patients with primary biliary cirrhosis that have a biochemical response to ursodeoxycholic acid. Gastroenterology. 2009;136(4):1281-7.

12. Farnaud S, Evans RW. Lactoferrin-a multifunctional protein with antimicrobial properties. Mol Immunol. 2003;40(7):395-405.

13. Berlutti F, Morea C, Battistoni A, Sarli S, Cipriani P, Superti F, et al. Iron availability influences aggregation, biofilm, adhesion and invasion of Pseudomonas aeruginosa and Burkholderia cenocepacia. Int J Immunopathol Pharmacol. 2005;18(4):661-70.

14. Actor JK, Hwang S-A, Kruzel ML. Lactoferrin as a natural immune modulator. Curr Pharm Des. 2009;15(17):1956-73.

15. Ishikado A, Imanaka H, Takeuchi $\mathrm{T}$, Harada E, Makino T. Liposomalization of lactoferrin enhanced it's anti-inflammatory effects via oral administration. Biol Pharm Bull. 2005;28(9):1717-21.

16. Legrand D, Elass E, Carpentier M, Mazurier J. Lactoferrin. Cell Mol Life Sci. 2005;62(22):2549-59.

17. Berlutti F, Pantanella F, Natalizi T, Frioni A, Paesano R, Polimeni A, et al. Antiviral properties of lactoferrin - $\mathrm{a}$ natural immunity molecule. Molecules. 2011;16(8):6992-7018.

18. Bharadwaj S, Naidu AGT, Betageri G V, Prasadarao N V, Naidu AS. Milk ribonucleaseenriched lactoferrin induces positive effects on bone turnover markers in postmenopausal women. Osteoporos Int. 2009;20(9):1603-11.
19. Gibbons JA, Kanwar RK, Kanwar JR. Lactoferrin and cancer in different cancer models. Front Biosci - Sch. 2011;3 S(3):1080-88.

20. Heinrich S, Georgiev P, Weber A, Vergopoulos A, Graf R, Clavien P-A. Partial bile duct ligation in mice: A novel model of acute cholestasis. Surgery [Internet]. 2011;149(3):44551. Available from: https://www.sciencedirect.com/science/article/pii/ S0039606010004149

21. Farid AS, El Shemy MA, Nafie E, Hegazy AM, Abdelhiee EY. Anti-inflammatory, antioxidant and hepatoprotective effects of lactoferrin in rats. Drug Chem Toxicol [Internet]. 2021;44(3):286-93. Available from: https://doi.org/10.1080/01480545.2019.1585868

22. Cooper DM, Hoffman W, Tomlinson K, Lee H-Y. Refinement of the dosage and dosing schedule of ketoprofen for postoperative analgesia in Sprague-Dawley rats. Lab Anim (NY) [Internet]. 2008;37(6):271-5. Available from: https://doi.org/10.1038/laban0608-271

23. Uchiyama M, Mihara M. Determination of malonaldehyde precursor in tissues by thiobarbituric acid test. Anal Biochem. 1978;86(1):271-8.

24. Nishikimi M, Rao NA, Yagi K. The occurrence of superoxide anion in the reaction of reduced phenazine methosulfate and molecular oxygen. Biochem Biophys Res Commun. 1972;46(2):849-54.

25. Marcellin P, Kutala BK. Liver diseases: A major, neglected global public health problem 
requiring urgent actions and large-scale screening.

Liver Int. 2018;38:2-6.

26. Mariotti V, Strazzabosco M, Fabris L, Calvisi

DF. Animal models of biliary injury and altered bile acid metabolism. Biochim Biophys Acta (BBA)-Molecular Basis Dis. 2018;1864(4):125461.

27. Yin H, Cheng L, Holt M, Hail N, MacLaren $\mathrm{R}$, Ju C. Lactoferrin protects against acetaminophen-induced liver injury in mice. Hepatology. 2010;51(3):1007-16.

28. Yin H, Cheng L, Agarwal C, Agarwal R, Ju C. Lactoferrin protects against concanavalin Ainduced liver injury in mice. Liver Int. 2010;30(4):623-32.

29. Sharawy MH, Abdel-Rahman N, Megahed N, El-Awady MS. Paclitaxel alleviates liver fibrosis induced by bile duct ligation in rats: Role of TGF$\beta 1$, IL-10 and c-Myc. Life Sci [Internet]. 2018;211(September):245-51. Available from: https://doi.org/10.1016/j.lfs.2018.09.037

30. Chang ZY, Chen CC, Liu HM, Yeh YC, Lin TY, Lee TY, et al. Positive effects of Ger-GenChyn-Lian-Tang on cholestatic liver fibrosis in bile duct ligation-challenged mice. Int $\mathrm{J}$ Mol Sci. 2019;20(17):1-12.

31. Zong Y, Zhang M, Li S, Qi W, Li J, Liu T, et al. Effects of Ethyl Pyruvate on Bile Duct LigationInduced Liver Fibrosis by Regulating Nrf2 Pathway and Proinflammatory Cytokines in Rats. Gastroenterol Res Pract. 2019;2019:1-11.

32. Ramos-Gomez M, Kwak MK, Dolan PM, Itoh K, Yamamoto M, Talalay P, et al. Sensitivity to carcinogenesis is increased and chemoprotective efficacy of enzyme inducers is lost in nrf2 transcription factor-deficient mice Sci. U.S.A., 98, 3410-15 (2001).

33. Cao Y-W, Jiang Y, Zhang D-Y, Wang M, Chen W-S, Su H, et al. Protective effects of Penthorum chinense Pursh against chronic ethanolinduced liver injury in mice. J Ethnopharmacol. 2015;161:92-8.

34. Surh Y-J. Cancer chemoprevention with dietary phytochemicals. Nat Rev Cancer. 2003;3(10):768-80. 